\title{
OCTAVIANUS UND DER TOTENGEIST DES IULIUS CAESAR IN DER RÖMISCHEN GESCHICHTE DES FLORUS
}

\begin{abstract}
Summary: The contribution investigates the references of the ghost of Iulius Caesar in Florus II 16, with the help of textual analogies. The references are analysed from the point of view of ancient historiography and religious history, examining the role of the deceased Iulius Caesar as prodigium (not as divus Iulius) in the Augustan propaganda.
\end{abstract}

Key words: ghosts, history writing, exempla, Augustan propaganda, second triumvirate, prodigium

In der sog. Epitome des Geschichtsschreibers Florus liest man im Buch II, caput 16 bezüglich des Konflikts von Perusia über die verschiedenen Motivationen der Mitglieder des zweiten Triumvirats im Jahre 43 und über die Teilnahme Octavians an dem blutigen Triumvirat als Zwangsentscheidung, um einen noch blutigeren Bürgerkrieg zu vermeiden:

Cum solus etiam gravis paci, gravis rei publicae esset Antonius, quasi ignis incendio Lepidus arcessit. Quid contra duos consules, duos exercitus? Necesse fuit venire in cruentissimi foederis societatem. Diversa omnium vota, ut ingenia. Lepidum divitiarum cupido, quarum spes ex perturbatione rei publicae, Antonium ultionis de qui se hostem iudicassent, Caesarem inultus pater et manibus eius graves Cassius et Brutus agitabant. ${ }^{1}$

Das Hauptziel des Lepidus sei nach der Darstellung des Florus die Erhöhung seines Vermögens, Antonius wolle Rache an den Senatoren nehmen (z.B. an Cicero), die ihn als Feind des Staates angeklagt haben. Octavianus wird inzwischen von seinem

\footnotetext{
${ }^{1}$ Florus II 16 (IV 6) 1-2 Havas.
} 
ungerächten Vater, Iulius Caesar, getrieben. Nach der Formulierung des lateinischen Satzes bleibt eine grundsätzliche Frage offen: Handelt es sich um den Totengeist des Caesars, der als ahoros keine Ruhe mehr in seinem Grab finden kann, ${ }^{2}$ solange er inultus ist? Oder ist er nur noch ein mehr oder weniger scharfes Schattenbild in der Erinnerung seines Adoptivsohnes, der wegen der necessitas pietatis erga parentem selbst keine Ruhe finden kann, solange er keine Rache nimmt?

Vielleicht hilft der vorige Satz: diversa omnium vota, ut ingenia. Laut Florus ist avaritia die dominante Motivation des Lepidus, ultio des Antonius und wieder ultio des Octavianus. Nach der augusteischen Propaganda kümmert sich aber Octavian überhaupt nicht um seine eigenen Interessen bzw. um die Verstärkung seiner eigenen Macht. Umso mehr kümmert er sich um die pietas erga patrem, die Treibkraft seiner eigenen Motivationen, unbedingt Rache zu nehmen, aber - wie Florus später in demselben caput mit Befangenheit schreibt - Octavian strebt während der blutigen Proskription vorwiegend die Verfolgung der Caesarmörder an. Als Motivation wurde pietas auch in dem kurzen Kommentar dieser Stelle von Luigi Bessone betont. ${ }^{3}$ Die vota, das Gelübde der einzelnen Triumviren erscheint in dem Text als Objekt der Motivationen, die Rede ist somit nicht unbedingt über eine explizite Totengeist-Erscheinung.

Da das lateinische Wort manes im Text mit einem Personalpronomen begleitet ist (manes eius), handelt es sich in diesem Fall nicht um eine allgemeine Bezeichnung des Totenreichs, sondern um den Totengeist Caesars selbst. ${ }^{4}$ Diese Interpretation bekräftigt auch die Benennung des Subjekts des Satzes: Caesar agitiert Octavianus als inultus pater, ebenso wie Brutus und Cassius ihn agitieren, die der Seele Caesars nicht mehr leicht werden können, also - wenn Florus hier auf die Trauerformel sit tibi terra levis anspielt - kann die Erde dem Caesar wegen des Attentats, wegen seinem voreiligen Tod nicht mehr leicht werden. Caesar scheint daher in diesem Text kein Gott, kein Subjekt des sidus Julium zu sein, ${ }^{5}$ sondern nur eine arme Seele, ein

${ }^{2}$ Nock, A. D.: Tertullian and the Ahori. In Nock, A. D.: Essays on Religion and the Ancient World. Ed. J. STEWART. Oxford 1972, 712-719; WASzINK, J. F.: Mors immatura. In WASZINK, J. F.: Opuscula selecta. Leiden 1979, 114-116; NAGY, L.: Die Typen von Totengeistern in der De anima von Tertullian und in der frühchristlichen Literatur. Specimina Nova 20 (2006) 13-14; OGDEN, D.: Greek and Roman Necromancy. Princeton 2004, 225-226.

${ }^{3}$ Bessone, L.: Ottaviano, Augusto e il regnum dei Caesares. ACD 40-41 (2004-2005) 314-315.

${ }^{4}$ Neuere religionsgeschichtliche Bewertungen zu den Gestalten der Dii Manes bezüglich der frühkaiserzeitlichen Vorstellungen über Totengeister s. LINDSAY, H.: The Romans and Ancestor Worship. In Religion in the Ancient World. New Themes and Approaches. Ed. J. DiLlon. Amsterdam 1996, 274-275; NAGY, L.: Zurückkehrende Toten und Gespenster-Liebe. Parallelen zweier kaiserzeitlichen Grabinschriften in der griechisch-römischen Literatur. In Epigraphica II, Mensa Rotunda epigraphiae Daciae Pannoniaeque. Eds. Gy. NÉMETH - I. PISO [Hungarian Polis Studies 11]. Debrecen 2004, 62-63, 70-72; KolB, A. - Fugmann, J.: Tod in Rom. Grabinschriften als Spiegel römischen Lebens. Mainz 2008, 1112; WitTEYeR, M.: Dis Manibus - den Totengöttern. In Götterbilder-Menschenbilder: Religion und Kulte in Carnuntum. Hrsg. F. HuMER - G. KREMER. Wien 2011, 75-77; NAGY, L.: Ancient Greek and Roman Ghost Stories. Some New Approaches. In Angels, Demons and Representations of Afterlife within the Jewish, Pagan and Christian Imagery. Coord. I. MoGA. Iaşi 2013, 228-229.

${ }^{5}$ Eine maßgebende Zusammenfassung über die Forschungsprobleme im Bereich der Interpretationen der Caesarapotheose aus der Sicht der Entstehung des römischen Kaiserkults s. GRADEL, I.: Emperor Worship and Roman Religion. Oxford 2002, 54-72. 
armer Geist, der nur auf die Rache, auf das Zeichen der Pietas seines Adoptivsohnes wartet. ${ }^{6}$ Hier muss man annehmen, dass pietas auch in diesem Sinne als eine korrekte Beziehung zu der Götter- und Geisterwelt interpretiert werden muss, wie unlängst John Scheid in seinem Artikel über die augusteische Religion formulierte, ${ }^{7}$ also die pietas des Octavianus gegenüber seinem Adoptivvater spiegelt auch nicht unbedingt seine persönlichen religiösen Ansprüche wieder.

Das Geschichtswerk des Florus wurde von der Mehrheit der Forschung entweder auf die Zeit Hadrians oder Antoninus Pius datiert. ${ }^{8}$ Vor einigen Jahren wurde von José Miguel Alonso-Nuñez auch eine Datierungsmöglichkeit auf das Ende der Regierungszeit Traians, um die Jahre 116 oder 117 bis zu den ersten Jahren Hadrians erwogen. ${ }^{9}$ Im Jahre 1994 hat Karl August Neuhausen merkwürdige und begründete Argumente für eine Frühdatierung des ganzen Geschichtswerks kurz nach dem Tode des Augustus erörtert, durch eine sorgfältige philologische Analyse konnte er sogar die Interpolationen der späteren Neuauflagen hadrianischer Zeit identifizieren. ${ }^{10}$ Er erkannte den Autor des römischen Geschichtswerks in Julius Florus, an den Horaz zwei Briefe (I 3 und II 2) richtete. ${ }^{11}$

Nach mehreren Artikeln von László Havas über Florus war die augusteische Zeit wirklich eine Erfüllung, eine gewisse Krönung der römischen Geschichte für den untersuchten Geschichtsschreiber. ${ }^{12}$ Die Ereignisse des zweiten Triumvirats führen den Leser in die Richtung einer zunehmenden climax der Geschichte, wie Luigi Bessone formulierte, ${ }^{13}$ wie die Macht zunehmend in die Hand eines einzelnen Politikers geriet. Ein wichtiger Punkt in diesem Prozess war nach der Darstellung des Florus das Abkommen des zweiten Triumvirats, denn laut Florus, caput 15 des zweiten Buches agierte Octavian vor 43 vorwiegend als Privatperson, z.B. im Konflikt von $\mathrm{Mu}-$ tina. Der Totengeist von Caesar als Motivation der Rache, die Octavian in den Triumvirat zwingt, ist deshalb auch ein wichtiger Punkt im Entfaltungsprozess der Geschichte, die in die Richtung des augusteischen Prinzipats führt. Die Darstellung von Caesars Seele zugleich als Totengeist und als göttliches Wesen ist keine unbekannte

${ }^{6}$ NAGY, L.: Kisértetek és kisértethit a kora császárkori történetírásban és politikai költészetben [Totengeister und Totengeister-Vorstellungen in der frühkaiserzeitlichen Geschichtsschreibung und politischen Dichtung]. Ungedr. Ph.D. Diss. Debrecen 2002, 130; NAGY, L.: Totengeister in der frühkaiserzeitlichen Geschichtsschreibung. ActAntHung 43 (2003) 92.

${ }^{7}$ SCHEID, J.: Augustus and Roman Religion. Continuity, Conservation and Innovation. In The Cambridge Companion to the Age of Augustus. Ed. K. GALINSKY. Cambridge 2005, 176-177.

${ }^{8}$ Obwohl B. Baldwin die bisherige Florus-Forschung bereits im Jahre 1988 auf mehrere ungelöste Probleme, auch bezüglich der Datierung aufmerksam gemacht hat: BALDWIN, B.: Four Problems with Florus. Latomus 47 (1988) 134-142.

${ }_{9}$ AlONSO-NuÑEZ, J. M.: Floro y los historiadores contemporaneos. ACD 42 (2006) 118-119.

${ }^{10}$ NeUHAUSEN, K. A.: Der überhörte „Schwanengesang“ der augusteischen Literatur: eine Rekonstruktion der Originalfassung (um 15. n. Chr.) des bisher dem 2. Jahrhundert zugeordneten Geschichtswerkes des Florus. ACD 30 (1994) 149-207. Siehe dazu noch NEUHAUSEN, K. A.: Augustus und Florus vor 2000 Jahren: Zur Wiederentdeckung und Rekonstruktion der Originalfassung des Geschichtswerkes des Iulius Florus (14/15 n. Chr.) in diesem Band, pp. 317-356.

${ }^{11}$ NEUHAUSEN: Schwanengesang (Anm. 10) 203-207.

12 HAVAS, L.: Florus-problémák [Florus-Studien]. AntTan 41 (1997) 102; HAVAS, L.: Un pseudotriomphe d' Hadrien aux frontieres d' apres Florus. ActAntHung 40 (2000) 177-180.

${ }^{13}$ BESSONE (Anm. 3) 320. 
Erscheinung in der frühkaiserzeitlichen lateinischen Literatur und Geschichtsschreibung.

Im Buch I, caput 8 der Dicta et Facta Memorabilia des Valerius Maximus erscheint Caesars Geist dem Caesarmörder Cassius auf dem Schlachtfeld von Philippi: Der tote Caesar greift ihn auf einem Pferd reitend mit drohendem Gesicht an. Der böse Caesar sagt Cassius, er habe ihn nicht getötet, denn er verletzte nur noch den sterblichen Körper, ihn selbst als Gott nicht:

Facta mentione urbis e qua primordia ciuitas nostra traxit, Divus Iulius, fausta proles eius [Ascanii] se nobis offert. quem C. Cassius numquam sine praefatione publici parricidii nominandus, cum in acie Philippensi ardentissimo animo perstaret, vidit humano habitu augustiorem, purpureo paludamento amictum minaci uoltu et concitato equo in se impetum facientem. quo aspectu perterritus tergum hosti dedit, uoce illa prius emissa: „,quid enim amplius agam, si occidisse parum est?" non occideras tu quidem, Cassi, Caesarem, neque enim ulla extingui divinitas potest, sed mortali adhuc corpore utentem uiolando meruisti, ut eum infestum haberes deum. ${ }^{14}$

Der Senat ließ dem Caesar noch vor seinem Tod göttliche Ehrenmaßnahmen einführen, z.B. wurden ihm als hemitheos laut Formulierung des Cassius Dio in seinem Buch 43. 14. 6. seine Statuen im Tempel des Quirinus auf dem Capitolium aufgestellt und er erhielt den Ehrentitel divus Iulius. Trotzdem wurde er offiziell erst im Jahre 42 v. Chr., mit der Gründung seines Tempels am Forum Romanum als Gott verehrt; Antonius bekleidete das Amt des flamen Iulii erst im Jahre $40 .{ }^{15}$ Der Satz neque ulla exstingui divinitas potest ist eine verallgemeinernde Behauptung, die seine geschickte Rhetorik lobt. In einem anderen exemplum im Buch VI, caput 8 schreibt er über die Rache des göttlichen Iulius Caesar, die er durch Pindarus vollendete: Cassius bat Pindarus, ihn nach der verlorenen Schlacht zu töten.

Das Werk des Valerius Maximus datiert die communis opinio der altertumswissenschaftlichen Fachkreise auf die Zeit des Tiberius, zwischen 14 und 31/32 n. Chr. ${ }^{16}$ Obwohl Valerius Maximus in seinen Memorabilia das tyrannische Benehmen des Caesars und das traurige Schicksal der Opfer der Proskription des zweiten Triumvirats an mehreren Stellen behandelt, lobt er Caesar wegen seiner militärischen Tapferkeit und seiner clementia, die auch für Tiberius wichtige Tugenden waren. Der Prozess gegen Cremutius Cordus im Jahre $22 \mathrm{n}$. Chr. konnte Valerius Maximus daran erinnern, dass die Beschreibung des Bürgerkriegs immer noch ein peinliches Thema ist. ${ }^{17}$

\footnotetext{
${ }^{14}$ Val. Max. I 8. 8 Briscoe.

${ }^{15}$ Zur Bewertung dieses Prozesses s. neuerlich SCHEID: Augustus (Anm. 7) 178, 184; GRADEL (Anm. 5) 54-59, 61-69.

${ }^{16}$ S. WARDLE, D.: The "Sainted Julius". Valerius Maximus and the Dictator. Classical Philology 92.4 (1997) 327-333; Bellemore, J.: When did Valerius Maximus Write the Dicta et Facta Memorabilia? Antichthon 23 (1989) 67-80.

${ }^{17}$ WARDLE (Anm. 16) 328, 345.
} 
Valerius Maximus erwähnt Caesar an 35 Stellen, davon schreibt er 20-mal über die Gottheit Caesars mit den Termini deus, divus, divinitas, divinus, numen, sidus. Es ist kein Zufall, dass er in der vorliegenden Geistererscheinung auf dem Schlachtfeld von Philippi kein Wort wie manes, species oder imago verwendet, sondern nur deus. ${ }^{18}$

Das Wort species für die Erscheinung des toten Caesars wird in der vita Augusti von Suetonius verwendet: Auf dem Schlachtfeld von Philippi trat die species des divus Caesar einem Mann aus Thessalien auf einem abgelegenen, „straßenlosen“, d.h. „umwegsamen“ Weg (itinere avio) entgegen, um den zukünftigen Sieg in der Schlacht anzukündigen. Dieser Mann gab Octavian die günstige Nachricht weiter. Das Ereignis wird im Text des Suetonius in eine Reihe von fünf Prodigia geordnet, die alle einen Sieg des Augustus vorhergesagt haben: ${ }^{19}$

Philippis Thessalus quidam de futura victoria nuntiavit auctore Divo Caesare, cuius sibi species itinere avio occurrisset. ${ }^{20}$

Appian beschreibt in seiner Emphylia Buch II, caput 146 Caesars Bestattung, wo ein Chor ein Trauerlied (epikedeion) sang. In dem Lied wurde auch der tote Diktator personifiziert, als hätte er seine eigenen guten Taten in seinem Leben aufgelistet und sich deshalb über seinen unerwarteten Tod gewundert. ${ }^{21}$ István Borzsák suchte die Quelle dieser Beschreibung in den Historiae von Asinius Pollio, die den frühkaiserzeitlichen Geschichtsschreibern auch bekannt gewesen sein könnte. ${ }^{22}$ Somit könnten auch die vorliegenden Geschichten bezüglich der Erscheinung Caesars nach seinem Tod die fiktive Erscheinung und den Monolog des toten Caesars auf der Bühne inspiriert haben.

Ein gemeinsamer Punkt dieser Geschichten ist die Erscheinung des toten Caesars als prodigium, ${ }^{23}$ das sich entweder mit dem Untergang der Caesar-Mörder oder mit der Vorhersage des Sieges bei Philippi verbindet. Laut der Georgica von Vergil, Buch ${ }^{24}$ und der Metamorphoses des Ovid, Buch XV ${ }^{25}$ wird Caesars Tod mit ungünstigen Prodigien, darunter auch Geistererscheinungen, begleitet. Im Buch I der Pharsalia des Lucanus, ${ }^{26}$ sowie in dem Gedicht Bellum civile in dem Satyricon des

${ }^{18}$ WARDLE (Anm. 16) 336-344, Anm. 87.

${ }^{19}$ Gugel, H.: Studien zur biographischen Technik Suetons [Wiener Studien, Beiheft 7]. Aus dem Nachlaß hrsg. von K. VRETSKA. Wien-Köln-Graz 1977, 43-44.

${ }^{20}$ Suet. Aug. 96 Roth.

${ }^{21}$ App. Emph. 2146 (611).

${ }^{22}$ BORZSÁK, I.: Caesars Funeralien und die christliche Passion. ActAntHung 10 (1962) 23-31.

${ }^{23}$ Zum Konzept von prodigium als einem gewissen Signal eines Krisenzustands in der römischen Gesellschaft s. LiebeSChUETZ, J. H. W. G.: Continuity and Change in Roman Religion. Oxford 1979, 151; McBain, B.: Prodigy and Expiation: A Study in Religion and Politics in Republican Rome [Latomus Collection 177]. Bruxelles 1982, passim; SCHEID, J.: Le rite des auspices à Rome: Quelle évolution? Reflexions sur la transformation de la divination publique des romains entre le $\mathrm{III}^{\mathrm{e}}$ et le $\mathrm{I}^{\mathrm{e}}$ siècle avant notre ère. In La Raison des signes. Présages, rites, destin dans les sociétés de la Méditerranée ancienne. Religions in the Graeco-Roman World 174. Éd. par S. GEORGOUDI - R. KOCH - F. SCHMIDT. Leiden-Boston 2012, 109-128.

${ }^{24}$ Verg. Georg. I 467-480.

${ }^{25}$ Ovid. Met. XV 783-798.

${ }^{26}$ Luc. Phars. I 569-570. 
Petronius ${ }^{27}$ erscheinen die ungünstigen Prodigien, darunter auch Geister, während des Einzugs von Caesar als nefas in Rom, am Anfang des Bürgerkriegs mit Pompeius. ${ }^{28}$ Lucanus erwähnt nicht nur Geister, die unter der Führung einer Furie gegeneinander kämpfen, sondern auch den Geist von Sulla, der auf dem Marsfeld gesehen wurde, und den Geist von Marius, der bei dem Fluss Anio die dort arbeitenden Bauern erschreckte. ${ }^{29}$ In dem Prolog der Pharsalia ist auch der Geist des Crassus erwähnt, denn die Römer töten im Bürgerkrieg einander und nehmen keine Rache an den Parthern wegen seinem schamhaften Tod in Folge der Schlacht bei Carrhae. ${ }^{30}$ Die Vorbilder solcher Prodigien sind seit Franz Dornseiff im Buch III der Oracula Sibyllina bekannt, ${ }^{31}$ aber die Geister als ungünstige Prodigien knüpfen sich in den von mir untersuchten Texten fast ausschließlich an den Bürgerkrieg als nefas, unabhängig davon, ob Caesars Tod oder Caesars Diktatur als nefas-Ereignis dargestellt wurde.

Nach der Augustusbiographie von Sueton, caput 92 berücksichtigte Augustus in seinem Leben immer allerlei Prodigien, portenta et omina quaedem, wie Sueton formuliert, laut caput 90 hatte er riesige Angst vor Blitzen, ${ }^{32}$ so kann die Rede über den Geist von Caesar auch in der Geschichte von Florus als ein ungünstiges Prodigium interpretiert werden. Die Rache muss erfüllt und der Bürgerkrieg muss nach den nötigen Kriegen des Augustus beendet werden, damit die Weltordnung der römischen Welt wieder hergestellt wird.

\author{
Levente Nagy \\ Institut für Geschichtswissenschaft \\ Abteilung für Archäologie \\ Universität Pécs \\ Ungarn \\ nagy.levente@pte.hu
}

${ }^{27}$ Petr. Sat. 122 (Bell. civ. 137-138).

${ }^{28}$ S. dazu RUTZ, W.: Lucans Pharsalia im Lichte der neuesten Forschung. ANRW II 32.3 (1985)

1471.

${ }^{29}$ Luc. Phars. I 580-583.

${ }^{30}$ Luc. Phars. I 9-12.

${ }^{31}$ Or. Syb. III 787-804; DORNSEIFF, F.: Die sibyllinischen Orakel in der augusteischen Dichtung. In Römische Literatur der Augusteischen Zeit [Deutsche Akademie der Wissenschaften, Schriften der Sektion für Altertumswissenschaft 22. Hrsg. J. IRMSCHER - K. KUMANIECKI]. Berlin 1960, 46-50; s. noch NAGY: Die Typen von Totengeistern (Anm. 2) 32.

${ }^{32}$ Kurze Bewertung der Textstelle: SCHEID: Augustus (Anm. 7) 175-176. 